\title{
Teste de acuidade visual infantil realizado por estudantes de medicina: relato de experiência
}

\author{
Child visual acuity test performed by medical students: experience report
}

Prueba de agudeza visual infantil realizada por estudiantes de medicina: informe de la experiência

Gabriela Augusta Silva de Souza ${ }^{1}$, Eduardo Haddad Caleiro Garcia ${ }^{1}$, Gislaine Cristhina Bellusse ${ }^{1^{\star}}$.

\section{RESUMO}

Objetivo: Relatar a experiência de estudantes do terceiro semestre do curso de medicina acerca da realização do teste de acuidade visual, com a utilização da Escala Optométrica de Snellen, em escolares do bairro. Relato de Experiência: Trata-se de um relato de experiência baseado em atividade (teste de acuidade visual) desenvolvida no módulo do Programa de Integração de Ensino e Saúde da Família (PIESF). O teste foi realizado em maio de 2019, no período de contraturno escolar, de 19 escolares de 8 a 11 anos, de uma instituição de ensino de crianças vulneráveis socialmente, na periferia de uma cidade do interior do estado de São Paulo. O teste foi conduzido e interpretado pelos estudantes de medicina, supervisionados pela docente responsável pelo módulo, em uma das salas de aula da instituição. Considerações Finais: A experiência permitiu aos graduandos envolvidos a vivência da realidade enfrentada por vários profissionais do sistema público de saúde, bem como do trabalho em equipe quando foi possível o envolvimento de docentes e discentes do curso de medicina, professores e diretores da instituição de ensino e coordenação da UBS do bairro em questão além de ter possibilitado o aprimoramento da relação teoria-prática contribuindo para o enriquecimento acadêmico.

Palavras-chave: Acuidade visual, Prevenção de doenças, Promoção da saúde.

\begin{abstract}
Objective: to report the experience of the medical school undergraduate students attending the third semester, about performing the visual acuity test, using the Snellen Optometric Scale, in school-aged children in a specific neighborhood. Experience Report: This is an activity-based experience report (visual acuity test) developed in the module of the Integration Program for Teaching and Family Health. The test was carried out in May 2019, during the school shift period, of 19 school-aged children aged 8 to 11 years old, from an educational institution for socially vulnerable children, in the suburbs of a city located in countryside of the São Paulo State. The test was conducted and interpreted by medical undergraduate. students, supervised by the professor responsible for the module, in one of the institution's classrooms. Final Considerations: The practice allowed the students involved to experience the reality faced by several professionals from the public health system, as well as teamwork when it was possible to involve professors and undergraduate students of the medical , professors and directors of the educational institution and coordination of the Basic Health Unity of the specific neighborhood in question, in addition to enabling the improvement of the theory-practice relationship, contributing to academic enrichment.
\end{abstract}

Keywords: Visual acuity, Disease prevention, Disease promotion.

\section{RESUMEN}

Objetivo: Informar la experiencia de los estudiantes en el tercer semestre del curso de medicina sobre la realización de la prueba de agudeza visual, utilizando la Escala Optométrica de Snellen, en escolares del vecindario. Informe de Experiencia: Este es un informe de experiencia basado en actividades (prueba de agudeza visual) desarrollado en el módulo del Programa de Integración para la Enseñanza y la Salud Familiar. La prueba se llevó a cabo en mayo de 2019, durante el período de turno escolar, de 19 escolares de 8 a 11 años, de una institución educativa para niños socialmente vulnerables, en las periferias de una ciudad en el interior de São Paulo. La prueba fue

${ }^{1}$ Universidade de Franca (UNIFRAN), Franca - SP. *E-mail: gislaine.bellusse@unifran.edu.br. 
realizada e interpretada por estudiantes de medicina, supervisados por el profesor responsable del módulo, en una de las aulas de la institución. Consideraciones Finales: La experiencia permitió a los estudiantes universitarios involucrados experimentar la realidad que enfrentan varios profesionales del sistema de salud pública, así como el trabajo en equipo cuando fue posible involucrar a profesores y estudiantes del curso de medicina, profesores y directores de la institución educativa, y la coordinación dela Unidad Básica de Salud del barrio, además mejorar la relación teoría-práctica, contribuyendo al enriquecimiento académico.

Palabras clave: Agudeza visual, Prevención de enfermedades, Promoción de enfermedades.

\section{INTRODUÇÃO}

Uma medida de assistência primária importante é a detecção de problemas visuais, uma vez que o nosso relacionamento com o mundo exterior se faz principalmente por meio da visão, ao passo que prejuízos no processamento visual acarretam deficiência significativas para desenvolvimento infanto-juvenil (TOLEDO CC, et al., 2010). Nota-se que de todos os processos sensitivos o ato de ver e correlacionar os estímulos do meio externo para obtenção de um conteúdo informativo é o mecanismo mais importante para a aquisição cognitiva e o processo de socialização (SILVA CM, et al., 2013).

Junior JC, et al., 2015 evidenciaram nos resultados de seu estudo que teve como objetivo avaliar o conhecimento dos professores sobre sinais e sintomas acerca dos problemas de visão em escolares com baixa acuidade visual. $O$ estudo identificou percepção insatisfatória pelos professores diante do fato deles não conseguirem relacionar os problemas percebidos a baixa acuidade visual. Os resultados desse estudo evidenciaram que $62 \%$ dos estudantes avaliados com baixa acuidade visual além de $75 \%$ dos estudantes possuírem outros sinais que apontassem para a baixa acuidade visual.

A acuidade visual reduzida em crianças que frequentam a escola tem como causa mais comum os erros de refração como hipermetropia, miopia e astigmatismo representando um grande desafio à saúde pública (CBO, 2019; SHEELADEVIS S, et al., 2019). A detecção precoce dos vícios de refração possibilita correção ou minimização, contribuindo para um melhor rendimento em escala global da criança em idade escolar (TOLEDO CC, et al., 2010).

Quando a criança inicia a vida escolar a dificuldade visual pode ser percebida com maior facilidade devido ao esforço necessário para o desenvolvimento das atividades. No entanto, o professor pode encontrar barreiras na identificação do aluno com baixa acuidade visual, visto que, os sintomas podem ser semelhantes aos que surgem em casos de timidez ou até mesmo desinteresse (SALUM TGB, et al., 2015). Atualmente, a tabela de Snellen é o método mais utilizado para medir a acuidade visual pois apresenta como vantagem o baixo custo de implementação e a possibilidade verídica de triagem contemplando a facilidade de aplicabilidade e replicabilidade do exame (VALVERDE CM, et al., 2016).

Nessa perspectiva, o teste de acuidade visual realizado com auxílio da Escala Optométrica de Snellen é um dos melhores indicadores da função visual, uma vez que não é necessário um alto nível de especialização do examinador, dispensa treinamento prolongado e não exige grandes esforços por parte dos pacientes, nem mesmo equipamentos sofisticados para sua execução e compreensão. Desse modo, caso haja uma verificação de possíveis suspeitas de problemas oculares deve-se providenciar um exame médico oftalmológico o mais breve possível. Assim, gastos maiores são poupados, ao passo que o custeio da oftalmologia pelo Sistema Único de Saúde (SUS) representa o terceiro maior orçamento por especialidade, ultrapassado apenas pelo da cardiologia e oncologia (RÉGIS-ARANHA LA, et al., 2017).

Atrelado a isso, há unanimidade entre os especialistas da área uma vez que a detecção precoce de problemas visuais se torna relevante, pois impedem problemas graves no futuro, contribuindo para a prevenção de danos permanentes à visão (RÉGIS-ARANHA LA, et al., 2017). Diante da análise desse contexto, faz-se necessário o combate junto da identificação pregressa das alterações visuais, visto que o pleno desenvolvimento da anatomia e funcionalidade visual demanda um tempo significativo, podendo chegar até aos sete anos de idade a criança (VALVERDE CM, et al., 2016). De acordo com Ribeiro GB, et al. (2015) essa descoberta, possibilita a contribuição analítica e epidemiológica desses impasses fornecendo aporte para elaboração de um planejamento específico de saúde pública atrelada doenças visuais.

REAS / EJCH | Vol.12(12) | e4888 | DOI: https://doi.org/10.25248/reas.e4888.2020 Página 2 de 6 
Para Biberg-Salum TG, et al. (2015) as possíveis alterações visuais raramente são percebidas no âmago familiar anterior ao ingresso à vida escolar, uma vez que o ambiente doméstico pode não ser retratado nem percebido esse comprometimento. Desse modo, na maioria das vezes, tais desordens serão percebidas e possivelmente manejadas no ambiente escolar, juntamente com profissionais da atenção primária de saúde, pois é no mundo no aprendizado que a criança é inserida integralmente, demandando suas competências sociais e fisiológicas da sensopercepção, envolvendo, assim, todos os seus sentidos visuais. Portanto, se for insatisfatória, o desempenho escolar pode ser prejudicado a médio e longo prazo.

Não raro, na perspectiva de Neto CA, et al. (2014) a visão é formadora das várias esferas que compõe a criança como um ser social e posteriormente da sua atuação no tecido social frente a futura vida adulta. Ao passo que as consequências que não forem tratadas corretamente diante da baixa acuidade visual afetam não apenas o rendimento escolar infantil, mas o comportamento social na vida adulta, como acidentes trabalhistas, podendo culminar em um elevado encargo socioeconômico do país mediante tal situação, além do prejuízo na realidade do indivíduo.

A visão é tida como espelho e reflexo da composição individual e atuação do ser na sociedade, refletindo a sua importância frente ao pleno desenvolvimento infantil permeado pelas habilidades que tais órgãos do sentido permitem alcançar e executar. Portanto, não obstante, a identificação das dificuldades de aprendizagem em ambiente escolar relacionadas a baixa acuidade visual não seja tarefa fácil, é possível de ser alcançada reforçando o elo da educação em saúde, com a participação de uma equipe multidisciplinar aliada ao primeiro nível de atenção da saúde (NETO CA, et al., 2014).

Os objetivos do desenvolvimento sustentável da agenda 2030 (ODS 2030) contemplam 169 metas que deverão ser cumpridas até o ano de 2030. Dentre essas metas, destaca-se a garantia de ensino primário e secundário de qualidade com resultados de aprendizagem eficazes (ONU, 2015). Nesse sentido, surgiu a inquietação sobre como estaria a saúde visual das crianças do bairro onde desenvolvemos nossas atividades práticas no curso de medicina, na busca de uma articulação com um aprendizado eficaz. Com base nesse questionamento, este estudo teve como objetivo relatar a experiência de estudantes do terceiro semestre do curso de medicina acerca da realização do teste de acuidade visual, com a utilização da Escala Optométrica de Snellen, em escolares do bairro.

\section{RELATO DE EXPERIÊNCIA}

Trata-se de um relato de experiência baseado em atividade (teste de acuidade visual) desenvolvida no módulo do Programa de Integração de Ensino e Saúde da Família (PIESF). O teste de acuidade visual foi realizado em maio de 2019, no período de contraturno escolar, de 19 escolares de 8 a 11 anos, de uma instituição de ensino de crianças vulneráveis socialmente, na periferia de uma cidade do interior do estado de São Paulo.

O teste foi conduzido e interpretado pelos estudantes de medicina, supervisionados pela docente responsável pelo módulo, em uma das salas de aula da instituição. Com a finalidade de uma execução correta e eficiente, foi realizado o preparo do local e utilizou-se a Escala Optométrica de Snellen (adaptada para 3 metros), oclusor ocular, fita para demarcação da distância correta, um lápis de cor preta que foi utilizado para apontar os optotipos, impressos para anotação dos resultados e de encaminhamento ao especialista. Além disso, houve a orientação aos escolares frente a realização do teste, bem como a realização desse com lentes corretivas, caso a criança já fizesse o uso dos óculos. A escassez de iluminação das salas foi um empecilho inicial, mas foi contornado posteriormente.

Constatou-se que dentre os 19 alunos analisados, 6 necessitavam de encaminhamento a um oftalmologista (31,5\%). À medida que o teste obtivesse resultado igual ou abaixo de 0,7 ou $70 \%$, uma investigação mais profunda seria necessária, ao passo que o teste de acuidade remete apenas a uma triagem e não a um diagnóstico. Foram coletados os dados necessários para agendamento de consulta oftalmológica, junto à Unidade Básica de Saúde (UBS) do bairro para os educandos selecionados no processo de triagem. Posteriormente, o corpo administrativo da UBS em vigência relatou o comparecimento deles, demonstrando efetividade na ação de promoção da saúde atrelada aos princípios do manejo da atenção primária. 


\section{DISCUSSÃO}

A realização do teste em escolares na comunidade contribuiu para o desenvolvimento de ambas as partes, ou seja, de um lado os escolares do bairro pela avaliação da acuidade visual realizada e, do outro lado, os estudantes de medicina quando foi possível o desenvolvimento de habilidades técnicas específicas, bem como de comunicação, encorajados pela metodologia ativa proposta pelo curso, a qual proporciona a inserção dos estudantes em cenários práticos desde o primeiro semestre.

Além disso, favorecer a análise crítica diante do manejo do processo é relevante para os discentes de medicina. As condições ideais do teste nem sempre são fornecidas pela estrutura vigente no local. Dessa forma, o estímulo à improvisação para otimizar ao máximo tais circunstâncias faz-se no futuro médico o conceber de habilidades criativas e aumenta a confiança em cenários que requisitam maior poder de decisão.

Ressalta-se que, as habilidades criativas são desenvolvidas em todos os módulos práticos do PIESF uma vez que desenvolvemos nossas habilidades em muitos ambientes não controlados, como, por exemplo, escolas, praças, creches, centro de idosos, entre outros.Foi necessário, durante a execução do teste, a adaptação do espaço cedido, visto que ele estava exposto a muitos ruídos que poderiam interferir diretamente nos resultados coletados. Diante disso, houve troca do espaço para que a atividade ocorresse de forma adequada.

Esses dados vão ao encontro de recomendações que apontam que para uma boa execução do teste, deve-se considerar o preparo do local, que deve ser calmo, bem iluminado e sem ofuscamento. $O$ ideal é que a luz incida por trás ou dos lados da pessoa avaliada, evitando que ilumine diretamente a Escala de Sinais de Snellen (BRASIL, 2016).

Após a análise dos resultados, foi possível identificar que de todos os escolares encaminhados ao oftalmologista, mais da metade necessitou de lentes corretivas, dados que corroboram com estudo que aponta a necessidade de inclusão da avaliação oftalmológica nas estratégicas públicas, visto que, quanto mais precoce essa avaliação for realizada, melhor será o prognóstico dos envolvidos. Essas ações podem ser justificadas pela prevalência de baixa acuidade visual em escolares (BECKER TOF, et al., 2019).

Ressalta-se que cerca de $80 \%$ dos casos de deficiência visual podem ser evitados (WHO, 2013). Os dados do nosso estudo corroboram dados evidenciados no Brasil quando aproximadamente $20 \%$ dos escolares apresentam problemas visuais não corrigidos e ainda, 100 crianças a cada mil educandos possuem erros de refração com necessidade de óculos para minimizar esse problema (BRASIL, 2016).

A percepção e o conhecimento do ambiente acontecem desde o nascimento e permeia por toda a vida, sendo a visão o sentido que se encarrega dessa ação. A presença de qualquer lacuna nesse sentido, principalmente na infância, pode acarretar atrasos no desenvolvimento da criança devido a integração neuropsicomotora e perceptiva (CARVALHO KM, et al., 2016).

Frente a discussão abordada por Laignier MR, et al. (2010) foi estimado que a grande maioria das crianças brasileiras na idade escolar nunca passou por um exame oftalmológico. Diante disso, o ambiente escolar como uma instituição aglutinadora de muitas crianças, torna-se um ambiente altamente favorável para a aplicação do teste de acuidade visual nessa fase de alto desenvolvimento intelecto-social do indivíduo.

Analisando esse contexto, fica evidente que a essência do Programa de Integração de Ensino em Saúde da Família foi concluída, ao passo que a visita até a escola trouxe uma melhora na qualidade de vida dessas crianças em todas as suas esferas sociais, incluindo o âmbito da aprendizagem.

Em Silva CM, et al. (2013), os alunos com déficit visual apresentaram notas de matemáticas abaixo dos que não apresentavam déficit, concretizando, portanto, a necessidade de intervenção rápida e efetiva para correção das causas visuais. Evidencia-se a escola, no contexto da atenção primária, ambiente estratégico e oportuno para o alcance de metas por meio das ações educativas em saúde (BURNETT AM, et al., 2018).

Em seu estudo, Fernandes LA; Franzoi MAH (2020) enfatizam que a triagem no âmbito da saúde ocular realizada nas escolas consiste em uma estratégia sensível e custo efetiva, em que um de seus principais objetivos, aliada a atenção primária, é a redução da morbidade infantil, principalmente em áreas carentes de recursos socioeconômicos.

REAS / EJCH | Vol.12(12) | e4888 | DOI: https://doi.org/10.25248/reas.e4888.2020 Página 4 de 6 
Em seu estudo, Silva CM, et al. (2013), evidenciaram que a implementação de programas que detectam baixa acuidade visual e consequente prevenção de enfermidades oftalmológicos nos países desenvolvidos desencadeou uma diminuição brusca em relação aos custos de ações que representa o atendimento dos portadores de distúrbios oculares. Nessa perspectiva, Neto CA, et al. (2014) citam em seu estudo a campanha "Olho no Olho" realizada pelo Conselho Brasileiro de Oftalmologia juntamente com o Governo Federal na década de 90 , que tem por objetivo a triagem ocular em escolares, bem como a concessão de uma consulta e fornecimento de óculos.

Contudo, apesar de abranger grande parte da população, a campanha trouxe, em seu âmago, a dificuldade de aplicação, fundamentada pela escassez de examinadores prontos e seguros. Dessa forma, faz-se necessário um olhar mais crítico perante a acuidade visual de escolares, diminuindo os prejuízos tanto pessoais quanto na conjuntura governamental.

Prioritariamente, a carência de profissionais encarregados de prover a saúde ocular dos escolares pode ser suprida pelos discentes. Sendo assim, apesar da simples aplicabilidade do exame, faz-se necessário um reforço no treinamento para padronizar e otimizar os resultados. Além disso, ampliar a participação dos professores a fim de chegar a um bem comum para a saúde dos escolares, assunto abordado por Neto CA, et al. (2014) que referem atenção a alguns comportamentos dentro da sala de aula como dificuldade para ler, desenhar, copiar, aproximação exagerada do material aos olhos. Dessa forma, a participação do professor contribui diretamente para melhora ou resolução de distúrbios oculares e desempenho escolar.

Atrelado aos Objetivos de Desenvolvimento Sustentável (ODS), a essência do processo de triagem está contemplando a promoção de bem-estar social e a possibilidade de um processo de aprendizagem com condições mais eficazes. Uma vez que o teste de acuidade trará de forma indireta um maior ensejo às crianças que apresentam qualquer distúrbio visual, ao passo que após serem encaminhadas ao médico especialista poderá solucionar da melhor forma seu problema, evitando complicações maiores em seu desenvolvimento.

Contudo, essa experiência permitiu aos graduandos envolvidos a vivência da realidade enfrentada por vários profissionais do sistema público de saúde, bem como do trabalho em equipe quando foi possível o envolvimento de docentes e discentes do curso de medicina, professores e diretores da instituição de ensino e coordenação da UBS do bairro em questão. Além disso, o aprimoramento da relação teoria-prática contribuiu para o enriquecimento acadêmico diante do manejo biopsicossocial que permeia a atenção primária.

\section{REFERÊNCIAS}

1. BECKER TOF, et al. Avaliação da acuidade visual em escolares do ensino fundamental. Revista Brasileira de Oftalmologia, 2019; 78(1): 37-41.

2. BIBERG-SALUM TG, et al. Triagem para distúrbios visuais em escolares no município de Campo Grande, Mato Grosso do Sul, Brasil. Scientia Medica, 2015; 25(4):ID21389.

3. BRASIL. Ministério da Saúde e Ministério da Educação. Secretaria de Atenção à Saúde Departamento de Atenção Básica e Secretaria de Educação Básica. Cadernos Temáticos do PSE - Saúde Ocular. Brasília (DF): Ministério da Saúde e Ministério da Educação; 2016.

4. BURNETT AM et al. Interventions to improve school-based eye-care services in low-and middle-income countries: a systematic review. Bull World Health Organ, 2018.

5. CARVALHO KM et al. Prevenção da cegueira e deficiência visual na infância. Rio de Janeiro: Cultura Médica, 2016; p.52.

6. CONSELHO BRASILEIRO DE OFTALMOLOGIA. As condições de saúde ocular no Brasil 2019. São Paulo: 2019; $104 p$.

7. FERNANDES LA, FRANZOI MAH. Prevalência de baixa acuidade visual em crianças de uma escola da rede pública de ensino. Cogitare enferm, 2020;25: e67144.

8. JUNIOR JC, et al. Conhecimento de professores sobre sinais e sintomas indicativos de baixa acuidade visual em escolares. Revista de Enfermagem UFPE on line, 2015;9 (4): 7289-94.

9. LAIGNIER MR, et al. De olhos bem abertos: investigando acuidade visual em alunos de uma escola municipal de Vitória. Esc Anna Nery Rev Enferm, 2010; 14 (1): 113-19.

10. NETO CA, et al. Relação entre acuidade visual e condições de trabalho escolar em crianças de um colégio do ensino fundamental público de Curitiba. Revista Brasileira de Oftalmologia, 2014; 73 (4): 216-9.

11. ORGANIZAÇÃO DAS NAÇÕES UNIDAS (ONU). The Millennium Development Goals Report. Nova York, 2015.

12. RÉGIS-ARANHA LA, et al. Acuidade Visual e desempenho escolar de estudantes em um município na Amazônia Brasileira. Esc Anna Nery, 2017; 21(2): e20170032.

13. RIBEIRO GB, et al. Avaliação oftalmológica de crianças de escolas públicas de Belo Horizonte/MG: um panorama acerca da baixa acuidade visual. Revista Brasileira de Oftalmologia, 2015; 74(5): 288-91. 
14. SALUM, TGB, et al. Screnning for visual disturbances in schoolchildren in the city of Campo Grande, Mato Grosso do Sul, Brazil. Scientia Medica, 2015; 5(4); ID 21389.

15. SHEELADEVI S, et al. Prevalence of refractive errors, uncorrected refractive error, and presbyopia I adults in India: a systematic review. Indian Journal of Ophthalmology, 2019; 67 (5): 583-592.

16. SILVA CM, et al. Desempenho escolar: interferência da acuidade visual. Revista Brasileira de Oftalmologia, $2013 ; 72$ (3): 168-71.

17. TOLEDO CC, et al. Detecção precoce de deficiência visual e sua relação com rendimento escolar. Revista da Associação Médica Brasileira, 2010; 56(4): 415-9.

18. VALVERDE CM, et al. Detecção da prevalência de baixa visual e tratamento no grupo etário 4 a 7 anos. Revista Brasileira de Oftalmologia, 2016; 75(4): 286-9.

19. WORLD HEALTH ORGANIZATION (WHO). Universal eye health: a global action plan 2014-2019. Geneva: WHO, 2013. 